\title{
Validation and Refinement of a Steering Friction Increase Detection Algorithm Using Test Drive Data
}

\author{
Arash Mohtat*1, Graeme Garner ${ }^{2}$, Wen-Chiao Lin ${ }^{3}$, and Naser Mehrabi ${ }^{4}$ \\ ${ }^{1}$ Haply Robotics Inc., Montréal, QC, H2X 3A1, Canada \\ arash@haply.co \\ ${ }^{2,4}$ General Motors Company, Canadian Technical Centre, Markham, Ontario, L3R 4H8, Canada \\ graeme.garner@gm.com \\ naser.mehrabi@gm.com \\ ${ }^{3}$ General Motors Global R\&D, Warren, MI, 48092, USA \\ wen-chiao.lin@gm.com
}

\begin{abstract}
The Electric Power Steering (EPS) System provides steering assist in conventional vehicle driving and is the main actuator for vehicle lateral control in active safety features. Comprehensive EPS system fault diagnosis (i.e., anomaly detection, fault isolation, and fault quantification) has become increasingly important for the next generation of vehicles. Previous works have utilized computer simulation and hardwarein-the-loop experiments to develop early anomaly detection algorithms for electrical and mechanical failures in EPS systems. Using test drive data collected, this paper validates and refines a previously developed algorithm designed for detecting increases in EPS system internal mechanical friction. The data include 215 minutes of natural driving with different speeds and steering maneuvers. To illustrate robustness, noise factors, such as tire type, are also considered in the data collection. Issues of time delays and parameter uncertainty in the previous model-based algorithm have been alleviated in the new refined algorithm. This has been achieved by developing a Kalman filter-based joint state-parameter estimator. The new estimator uses a simplified vehicle dynamic model and provides a direct, and thus better, estimate of steering friction increase. Data collected from test drives indicate that the refined algorithm can robustly indicate a friction increase before an average human driver notices a difference in steering feel.
\end{abstract}

\footnotetext{
*Work documented in this paper was conducted while A. Mohtat was at General Motors Canadian Technical Centre.

Arash Mohtat et al. This is an open-access article distributed under the terms of the Creative Commons Attribution 3.0 United States License, which permits unrestricted use, distribution, and reproduction in any medium, provided the original author and source are credited.
}

\section{INTRODUCTION}

Electric Power Steering (EPS) systems have become commonplace in newer vehicles as a replacement to traditional hydraulic power steering systems (Badawy, Bolourchi, \& Gaut, 1997; Denton, 2004). In a typical steering maneuver, the EPS system compensates for a large portion of the steering torque required. In case of degradation or failure of the EPS system, it can instantly become very difficult to steer a vehicle, especially at slower speeds and could cause erratic driving behavior due to intermittent surprises for drivers. Moreover, the EPS system is the main actuator for vehicle lateral control in active safety systems. Consequently, active safety features would not function properly without a health EPS system. Advanced fault diagnosis (i.e., anomaly detection, fault isolation, and fault quantification) are critical to maintaining the functionality of the EPS system, and early fault / anomaly detection is a crucial prerequisite, thus the focus of this work.

Previous work related to advanced fault diagnosis of EPS systems include (Ji, Ge, \& Tian, 2013; Amberkar, Kushion, Eschtruth, \& Bolourchi, 2000; Lee, Lee, Kim, \& Jeong, 2007; Jeong et al., 2015; Huang \& Wang, 2013; Lin \& Ghoneim, 2016; Lin \& Du, 2018; Lawson \& Chen, 2008). In particular, (Ji et al., 2013) conducts a comprehensive design failure mode and effects analysis on EPS systems and identifies potential hazards in the system, and (Amberkar et al., 2000) develops requirements for diagnosis of EPS systems focusing on unwanted steer (i.e., failure that causes EPS to change direction of steering without a steering input). References (Lee et al., 2007) and (Jeong et al., 2015) develop algorithms that isolate sensor and motor failures in the EPS by comparing and cross checking estimated vehicle behavior, sensor 
measurements, and control commands. Detection of backlash in the steering system is developed in (Huang \& Wang, 2013), where a backlash model is recursively identified using estimates and measurements of road and hand wheel angles, respectively. The work in (Lin \& Ghoneim, 2016) applies model-based parameter estimation techniques to determine electric parameters of the EPS motor for fault / anomaly detection. In addition, changes in EPS mechanical parameters (i.e., anomalies such as friction increase) are detected by monitoring the deviation of self-aligning torque (SAT) estimated from two independent methods. Moreover, a fault signature table is constructed based on estimations of motor parameters, calculations of road SAT, and residuals of parity equations for EPS fault isolation. Fault / anomaly detection in power connection from the power source to the EPS, e.g., loose connectors or corrosion in the power wires, are considered in (Lin \& Du, 2018). Specifically, a canary-based method is proposed for detecting a loose connection, while power conservation laws are utilized to detect high resistance in an EPS power circuit. A fault-tolerant control strategy is developed in (Lawson \& Chen, 2008) to accommodate for driver input torque sensor failures by using an observer to estimate the total torque on the motor shaft and an independent estimate of the road reaction torque.

The current paper builds upon the work in (Lin \& Ghoneim, 2016) and uses new data collected under a large variety of injected faults and noise factors to validate and refine the steering friction increase detection algorithm. The ideas of Kalman filter-based joint state-parameter estimation documented in (Wan \& van der Merwe, 2001; Mohtat, Ghaffari Toiserkan, \& Kövecses, 2011; Mosquera Alonso, 2019) are used to improve estimates of the increased friction. As a result, an enhanced algorithm for detection of steering system friction increase is developed.

The rest of the paper is organized as follows. Section 2 provides a brief review of EPS systems and the friction fault considered, and Section 3 describes how friction is introduced in the test vehicle and the resulting test data. Section 4 reviews and validates the friction detection algorithm from (Lin \& Ghoneim, 2016) and develops the refined algorithm. Results of this refinement are shown in Section 5. Conclusions and future work are included in Section 6. For the sake of brevity, additional information regarding the levels of friction introduced for the test vehicle is summarized in Appendix A, and Appendix B explains how nonlinearity is treated in the joint state-parameter estimator presented here.

\section{EPS SYSTEMS AND INCREASED FRICTION FAULT}

EPS systems can be categorized based on where the EPS motor is mounted. For smaller vehicles, the EPS motor is usually mounted on the column and these EPS systems are hence referred to as column-mounted. For larger vehicles, the EPS motor has to be larger to provide adequate steering assist. In this case, the EPS motor is mounted on the steering rack. This kind of EPS system is referred to as rack-mounted. However, operations of the EPS systems are essentially the same. A column-mounted EPS system is shown in Figure 1. The

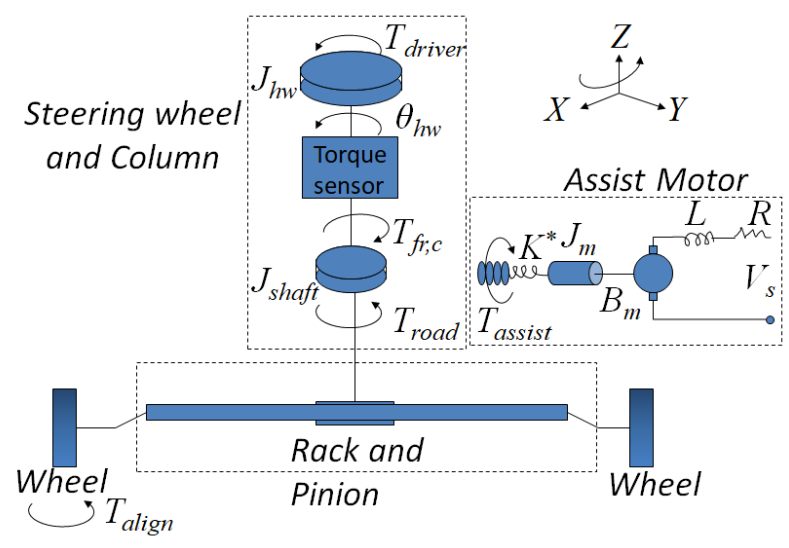

Figure 1. A column driven EPS system.

assist motor is attached to the steering column through a reduction gear box. The motor torque is applied to the steering shaft through the reduction gear and the product of the motor torque and the gear ratio produces a torque assist to the driver.

In this paper we consider detecting mechanical friction increase in the steering system, which may occur anywhere in the mechanical connections of the steering system from the steering column to the rack and pinion sub-component. An example is water intrusion into the rack and pinion mechanism causing corrosion and increased resistance against intended motion.

\section{Fault Injection and Vehicle Test data}

\subsection{Fault Injection}

In order to collect data for validating and refining developed friction detection algorithms, a test vehicle is instrumented for normal driving with various levels of steering friction. Specifically, friction faults are injected by installing a clutch brake on the EPS motor shaft to simulate added resistive torque from friction. The normal force applied to the clutch brake is applied by an electric motor with configurable Pulse Wave Modulation (PWM) varying from $0 \%$ to $100 \%$. The mapping between the PWM duty cycle setting of the clutch brake and the corresponding increase in friction is difficult to measure directly without a torque sensor on the EPS motor shaft. This mapping is approximated by studying the hysteresis loops of a lock-to-lock test with the instrumented vehicle, described in Appendix A. The fault levels (expressed as clutch brake modulation settings) and corresponding estimated friction increases are summarized in Table 1. The range listed in the table is the minimum/maximum estimated 
injected friction from 4 trials of hysteresis loop estimation. In order for a fault detection algorithm to be valid, it must be

\begin{tabular}{|l|c|c|}
\hline Fault level & Injected friction $(\mathrm{Nm})$ & Total friction $(\mathrm{Nm})$ \\
\hline Healthy $(\mathrm{H})$ & 0 & 4 \\
\hline PWM 15\% & $1-2$ & $5-6$ \\
\hline PWM 20\% & $3-5$ & $7-9$ \\
\hline PWM 25\% & $7-10$ & $11-14$ \\
\hline
\end{tabular}

Table 1. Steering friction fault injection values.

able to detect faults at or before the level at which a driver could notice them. Based on test drive experience, a normal driver does not notice a difference in steering feel until the total steering friction is increased to a level of $11-14 \mathrm{Nm}$. Hence, the objective is to develop a fault detection algorithm capable of detecting increased friction at or before the PWM $25 \%$ level of increased friction.

\subsection{Data Collection}

Since a high acquisition rate is required to execute the friction detection algorithm, test drive data was collected using a dSPACE micro autobox with acquisition rate of $10 \mathrm{~ms}$. Measured signals include those related to the steering system, such as EPS motor torque, driver torque, and steering angle, as well as vehicle dynamic signals such as angular rates and accelerations measured by an inertial measurement unit, and wheel speeds measured by encoders. No additional sensors were added to the test vehicle, as all needed signals are available in the production model. In each test, the increase in steering friction over the healthy system is approximated by the hysteresis loop estimation as summarized in Table 1 above. Table 2 below summarizes the quantity of data collected for each health state. Note that only development and

\begin{tabular}{|l|c|c|c|}
\hline Health state & $\begin{array}{c}\text { Change } \\
\text { (vs. Healthy) }\end{array}$ & $\begin{array}{c}\text { Development } \\
\text { set (min) }\end{array}$ & $\begin{array}{c}\text { Test set } \\
\text { (min) }\end{array}$ \\
\hline Healthy vehicle & NA & 98 & 78 \\
\hline Friction: PWM 15\% & +1 to 2 Nm & 7 & 6 \\
\hline Friction: PWM 20\% & +3 to 5 Nm & 7 & 6 \\
\hline Friction: PWM 25\% & +7 to $10 \mathrm{Nm}$ & 7 & 6 \\
\hline
\end{tabular}

Table 2. Friction increase detection algorithm test data.

test data sets are needed for the friction algorithm: the development set used for making initial design choices and tuning; and the test set to fairly assess the performance against unseen data. Due to the nature of the friction increase detection, we need long continuous streams of signals so we choose to focus on organic normal driving data. The organic normal drive tests are each about 13 minutes long with only one test per faulty health state resulting in the above distribution of test sets.

\section{Steering Friction Algorithm DeVelopment}

In this section, the performance of the friction detection algorithm presented in (Lin \& Ghoneim, 2016) is validated using vehicle test data, and iteratively refined to improve its performance. The original algorithm (as described in (Lin \& Ghoneim, 2016)) is referred to as the "Baseline Algorithm," and the refined version is referred to as the "Refined Algorithm."

\subsection{Baseline Algorithm}

In this subsection, we first give a brief review of the baseline algorithm. The central idea of the friction detection algorithm in (Lin \& Ghoneim, 2016) is to identify friction by comparing two independent estimates of the self-aligning torque (SAT, or $M_{z}$ ) acting on the vehicle's front wheels. Self-aligning torque is generated by the asymmetry of lateral force on the front tires' contact patch. Tire distortion under lateral force results in the average force applying at a distance $l_{p}$ from the contact patch's geometric center. This distance is known as the pneumatic trail and is approximately constant in the linear region of the tire dynamics (the region where slip angle and lateral force exhibit a linear relationship). The algorithm described in (Lin \& Ghoneim, 2016) identifies increased steering friction whenever the error between two SAT estimations grows large. The first SAT estimation method utilizes a balance of external forces on the vehicle, and the second SAT estimation method utilizes a balance of internal torques applied to the steering column. Since steering friction is an input to the second method but not the first, any change to the internal friction will present itself as an error between the estimations. The external SAT estimate $\left(M_{z}^{d y n}\right)$, which utilizes a bicycle model in the linear region of tire dynamics, is given by

$$
\begin{aligned}
M_{z}^{d y n} & =-\left(l_{p} C_{f} \frac{C_{f}}{C_{f}+C_{r}}\right) \rho(\theta) \theta \\
& -\left(l_{p} C_{f} \frac{M}{C_{f}+C_{r}}\right) a_{y}+\left(l_{p} C_{f} \frac{L C_{r}}{C_{f}+C_{r}}\left(\frac{\dot{\psi}}{v_{x}}\right)\right),
\end{aligned}
$$

where $C_{f}$ and $C_{r}$ are the front and rear tire cornering stiffness, respectively, $\theta$ is the steering wheel angle, $\rho(\theta)$ is the ratio between road wheel angle and steering wheel angle, $M$ is the vehicle mass, $L$ is the vehicle length, $a_{y}$ is lateral acceleration, $v_{x}$ is longitudinal velocity, and $\dot{\psi}$ is yaw rate (Lin $\&$ Ghoneim, 2016). The internal SAT estimate $\left(M_{z}^{o b s}\right)$ is estimated using a linear state observer (LSO), also known as a Luenberger Observer, applied to a model of the EPS motor system, governed by

$$
\begin{aligned}
\ddot{\theta} & =-\frac{B_{e q}}{J_{e q}} \dot{\theta}-\frac{T_{f}}{J_{e q}} \operatorname{sign}(\dot{\theta}) \\
& +\frac{T_{\text {driver }}-\rho(\theta) M_{z}^{o b s}}{J_{e q}}+\frac{K_{t} n}{J_{e q}} i
\end{aligned}
$$


where $\theta$ is the steering angle, $B_{e q}$ and $J_{e q}$ are, respectively, the damping and inertia constants of the steering system, $T_{f}$ is the nominal internal friction torque, $T_{\text {driver }}$ is the torque applied by the driver, $K_{t}$ is the EPS motor coefficient, $n$ is the EPS motor gear ratio, and $i$ is the EPS motor current (the control system input).

The friction state-of-health $(\mathrm{SOH})$ is inferred by comparing these two estimates of SAT. As internal friction increases above the nominal level, the observer estimate of SAT will also increase because the nominal friction used in the observer $T_{f}$ does not account for the friction increase. The $\mathrm{SOH}$ is inferred by calculating the error variance, $\operatorname{var}\left(M_{z}^{d y n}-\right.$ $\left.M_{z}^{o b s}\right)$, with larger variance in error indicating more friction. ${ }^{1}$ Figure 2 illustrates a concept of this baseline algorithm. As

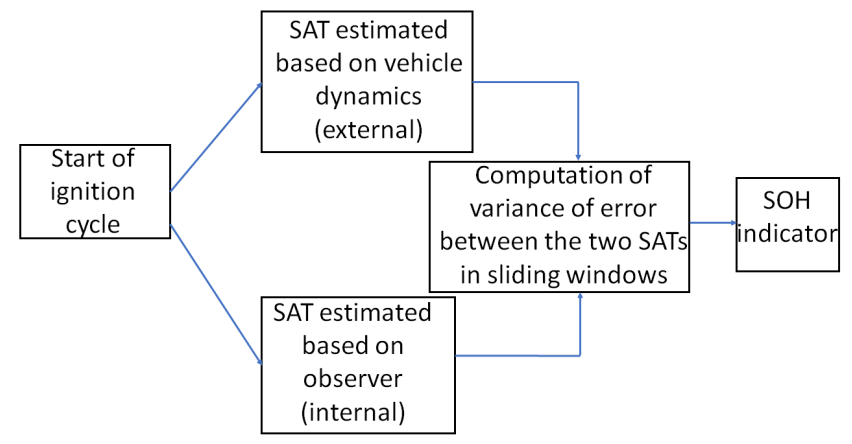

Figure 2. Concept diagram of baseline algorithm.

shown in Figure 2, a sliding window is used for calculating the error variance. The tuning parameters of this method are the cornering stiffness $\left(C_{f}, C_{r}\right)$, the pneumatic trail $\left(l_{p}\right)$, the observer gain matrix (Lin \& Ghoneim, 2016), and the size of window used to calculate the error variance.

\subsection{Baseline Algorithm Performance}

When the baseline friction fault detection algorithm presented in (Lin \& Ghoneim, 2016) was implemented on development set data, it was found to be difficult to distinguish between healthy and faulty steering systems. Various enabling conditions, rolling variance batch sizes, and signal alignment methodologies were experimented with to improve the results. The enabling conditions with the best performance were found to be requiring the steering angle to exceed $10^{\circ}$, the lateral acceleration to be between 0.1 and $0.5 \mathrm{~m} / \mathrm{s}^{2}$, and the slip angle between the front wheels and the road to be less than $5^{\circ}$. The average enabled ratio (i.e., the ratio of the data during which the algorithm is enabled) in the development set tests is $36 \%$. Figure 3 shows normalized histograms of $\mathrm{SOH}$ indicator values for healthy and faulty steering systems from the development data set. The overlap between the base-

\footnotetext{
${ }^{1}$ Compared to the absolute error, the variance of the error is more robust against the time-delay between the two $M_{z}$ estimations. See the discussion on refinement 1 in the next subsection for more details on the time-delay.
}

line algorithm outputs for healthy and faulty steering systems indicate that this $\mathrm{SOH}$ indicator does little to identify the friction fault. Although an exhaustive list is not included in this

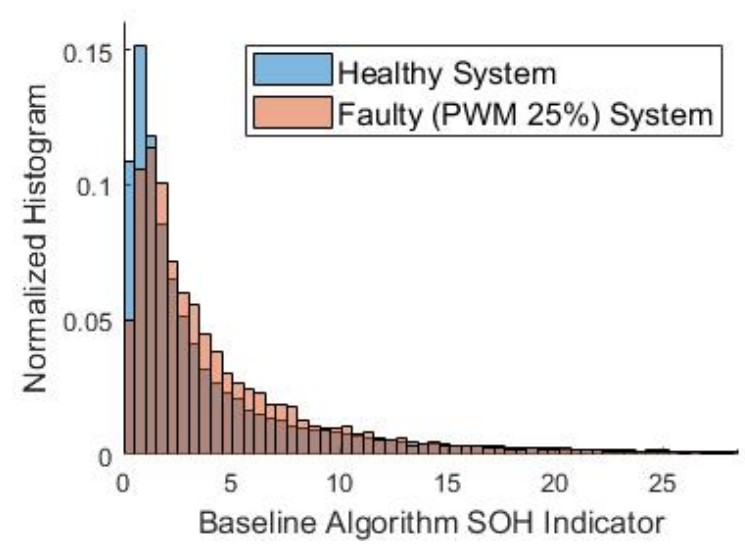

Figure 3. Normalized histogram of rolling error variances for healthy vs faulty (PWM 25\%) development data.

paper, enabling conditions and algorithm tuning parameters were explored thoroughly. The presented results summarize the best performing choices that were found.

\subsection{Refined Algorithm}

The steps taken to refine the outputs of the friction detection algorithm are summarized below, and the results of the refined algorithm with all implemented changes are presented in Section 5. While the identified refinements work in tandem to improve the algorithm performance, we can break down the motivation behind the changes in to three separate improvements. First, we identified the need to re-formulate the health indicator to a direct estimate of the friction torque. Second, we revised the vehicle dynamics model used to derive Eq. (1) to a slightly simpler model that allows us to reformulate the equations behind the separate SAT estimates as a joint state-parameter model. Finally, we improved the algorithm's ability to manage uncertainties and unknown dynamics by employing a Kalman Filter that offers the required tuning features.

Refinement 1: Direct Parameter Estimation. The baseline algorithm indirectly infers the friction state-of-health by computing the rolling variance of the error between two SAT estimates. While it is true that an increase in friction would lead to an increase in SAT error variance, there are many other factors that could cause an increase in SAT error. One significant source of error between the SAT estimates is the natural time-delay in the output of the observer used in the estimate of $M_{z}^{o b s}$. This time delay is caused by limitations on the data acquisition speed and the filtering nature of the observer. In particular, the internal SAT estimate $\left(M_{z}^{o b s}\right)$ lags both the steering angle signal and the external SAT estimate 
$\left(M_{z}^{d y n}\right)$. This signal delay introduces significant error between the estimates, making it challenging to derive an effective health indicator by comparing the two time-series, such as in $\operatorname{var}\left(M_{z}^{d y n}-M_{z}^{o b s}\right)$. We attempted to utilize signalshifting techniques such as dynamic time warping and crosscorrelation-based time realignment to improve the error variance calculation, but these methods did not yield any promising results. This analysis motivated the need to re-formulate the algorithm in a way that outputs a direct estimate of the steering friction.

Refinement 2: Bicycle Model Revision. The bicycle model used to derive Eq. (1) has two shortcomings: it is non-linear with respect to vehicle motion signals since speed and yaw rate appear in the same term; and, it uses a constitutive model that relates lateral tire forces to tire motion, i.e., slip angle. This not only adds calibration parameters that introduce complications in the tuning process, but also creates restrictions as the constitutive model is valid only in certain regimes of motion. To break free from these limitations, we refer to Figure 4 and use the following revised bicycle dynamics formulation:

$$
\begin{array}{r}
F_{y}^{\text {front }}+F_{y}^{\text {rear }}=m a_{y}, \\
L_{f} F_{y}^{\text {front }}-L_{r} F_{y}^{\text {rear }}=I_{z} \ddot{\psi}, \\
M_{z}=F_{y}^{\text {front }} l_{p},
\end{array}
$$

which implies

$$
M_{z}=\frac{l_{p}\left(L_{r} m a_{y}+I_{z}(\ddot{\psi})\right)}{L_{r}+L_{f}},
$$

where $F_{y}^{\text {front }}$ and $F_{y}^{\text {rear }}$ are lateral forces acting on the front and rear tires, $L_{f}$ and $L_{r}$ are the front and rear vehicle lengths from the center of mass, $m$ is the vehicle total mass, $I_{z}$ is the vehicle inertia about the $z$-axis, $l_{p}$ is the tire pneumatic trail, $a_{y}$ is lateral acceleration, $\dot{\psi}$ is yaw rate, and $M_{z}$ is the selfaligning torque. Note that the above equation has inverse dy-

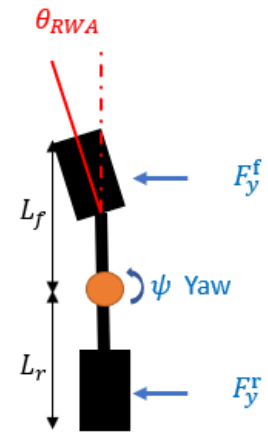

Figure 4. A simplified bicycle model.

namics causality, i.e., 2nd order motion derivatives are needed to calculate the torque $M_{z}$. In particular, the yaw acceleration term would make it difficult to use this equation directly to estimate $M_{z}$. Since direct differentiation of the yaw rate would introduce too much noise, such an equation had not been used in the baseline algorithm to provide an estimate of $M_{z}$. We solved this challenge by not using Eq. 4 in inverse dynamics mode for estimating $M_{z}$ in a silo; but, instead, using it in forward dynamics mode and in conjunction with other equations to formulate a multivariate joint state-parameter estimation problem as described next.

Refinement 3: Joint State-Parameter Estimation and Uncertainty Handling. In order to estimate friction using Eq. (2), we need to estimate $M_{z}$ using Eq. (4). However, we want to use the latter equation without resorting to direct numerical differentiation of the yaw rate. A creative way out of this dilemma is to regard the yaw rate as a state to be estimated and use Eq. (2) in the rearranged forward dynamics causality. This yields a 5 -variate problem with $\theta, \dot{\theta}, \dot{\psi}, M_{z}$ and $T_{f}$ to be estimated. However, so far we have only three equations (Eq. (2) counts as two due to its second-order nature). To balance the number of equations with the number of estimation variables, we regard the problem as a joint stateparameter estimation formulation and introduce virtual process dynamics into the picture. This is a well-known practice for constant parameters such as $T_{f}$. For the non-constant unknown state $M_{z}$, on the other hand, this is a novel, non-trivial design choice that we will refer to as a pseudo-parameter. In other words, we disguise a time-varying state as a parameterlike state governed by as a constant virtual process subject to a large virtual noise and estimate it simultaneously with the other variables. The linearized ${ }^{2}$ joint state-parameter formulation (5) below ties all the discussed refinements and novel design choices together. Note that the second row of the stateparameter matrix is the discrete-time adaption of Eq. (2); the third row is the discrete-time adaption of Eq. (4); and, the last two rows are the discrete-time descriptions of the selfaligning torque and friction as virtual pseudo-parameter and parameter processes:

$$
\begin{aligned}
& x_{t+1}=A_{t} x_{t}+B_{t} u_{t}+G_{t} \omega_{t} \\
& y_{t}=C_{t} x_{t}+v_{t},
\end{aligned}
$$

where

$$
x_{t}=\left[\begin{array}{c}
\theta_{t} \\
\dot{\theta}_{t} \\
\dot{\psi} \\
M_{z, t} \\
T_{f, t}
\end{array}\right], u_{t}=\left[\begin{array}{c}
T_{d, t} \\
T_{m, t} \\
a_{y, t}
\end{array}\right], \omega_{t}=\left[\begin{array}{c}
\omega_{T_{d}} \\
\omega_{T_{m}} \\
\omega_{a_{y}} \\
\omega_{M_{z}} \\
\omega_{T_{f}}
\end{array}\right], v_{t}=\left[\begin{array}{c}
v_{\theta} \\
v_{\dot{\theta}} \\
v_{\dot{\psi}}
\end{array}\right],
$$

\footnotetext{
${ }^{2}$ See Appendix B for a discussion on the existing nonlinearity and the linearization process.
} 


$$
\begin{aligned}
& A_{t}=\left[\begin{array}{ccccc}
1 & d t & 0 & 0 & 0 \\
0 & e^{-d t \frac{B_{e q}}{J_{e q}}} & 0 & -\beta \rho\left(\theta_{t}^{*}\right) & -\beta \operatorname{sign}\left(\dot{\theta}_{t}^{*}\right) \\
0 & 0 & 1 & d t \frac{L_{r}+L_{f}}{l_{p} I_{z}} & 0 \\
0 & 0 & 0 & 1 & 0 \\
0 & 0 & 0 & 0 & 1
\end{array}\right], \\
& G_{t}=\left[\begin{array}{ccccc}
0 & 0 & 0 & 0 & 0 \\
\beta & n \beta & 0 & 0 & 0 \\
0 & 0 & -d t \frac{L_{r} m}{I_{z}} & 0 & 0 \\
0 & 0 & 0 & d t & 0 \\
0 & 0 & 0 & 0 & d t
\end{array}\right] \\
& B_{t}=\left[\begin{array}{ccc}
0 & 0 & 0 \\
\beta & n \beta & 0 \\
0 & 0 & -d t \frac{L_{r} m}{I_{z}} \\
0 & 0 & 0 \\
0 & 0 & 0
\end{array}\right], \beta=\frac{1-e^{-d t \frac{B_{e q}}{J_{e q}}}}{B_{e q}}, \\
& C_{t}=\left[\begin{array}{lllll}
1 & 0 & 0 & 0 & 0 \\
0 & 1 & 0 & 0 & 0 \\
0 & 0 & 1 & 0 & 0
\end{array}\right] .
\end{aligned}
$$

Note that $d t$ is the discretization step size, e.g. $0.01 \mathrm{sec}$, and the terms with the ${ }^{*}$ superscript in $A_{t}$ are clarified in Appendix B.

The key element in making the virtual parameter and pseudoparameter description of $T_{f}$ and $M_{z}$ useful is the inclusion of their virtual process noises in the augmented process noise $\left(\omega_{t}\right)$ and make it play along with the measurement noise $\left(v_{t}\right)$, here modeled as zero-mean Gaussians with diagonal covariance matrices $Q$ and $R$, respectively. This allows a natural and powerful mechanism for managing and guiding uncertainty in our refined model: use of a Kalman Filter to estimate the joint state-parameter vector $x_{t}$ described as a stochastic process with multivariate noise properties. We extend the classic Kalman filter (Wan \& van der Merwe, 2001) by adding an enablement step that allows partial updating of the state vector depending on the inputs $u_{t}$ and measurements $y_{t}$. The enabling matrix $E_{t}$ is a diagonal matrix the same size as $A_{t}$, with 1 's in the positions of states that are to be updated, and 0 's in the positions of states that are held constant. The Enabled Kalman Filter is summarized in Algorithm 1 shown in Table 3.

The choice of virtual process noise (VPN) for the parameter and pseudo-parameter variables, i.e. $T_{f}$ and $M_{z}$, is critical to the Kalman filter's successful implementation. For the virtual pseudo-parameter, $M_{z}$, the VPN must be large so that the filter puts less trust in the constant virtual process dynamics and instead varies quickly based on each new measurement - effectively making the filter learn the unknown pseudo-parameter dynamics. This updating is driven by the connection between $\dot{\psi}$ and $M_{z}$ that is built in to the system via the $\dot{\psi}$ state equation (3). For $T_{f}$, the VPN must be relatively small to enable the system to learn and converge to the unknown constant parameter. ${ }^{3}$ In other words, by choosing a large VPN for the quickly changing $M_{z}$ pseudo-parameter variable and a small VPN for the slowly changing $T_{f}$ variable (supposed to learn a constant parameter), we ensure that most uncertainty is absorbed by the estimate of $M_{z}$. This in turn reduces the effect of model uncertainty on the estimate of $T_{f}$ and encourages it to converge to a narrow range of values. The health indicator outputted by this refined algorithm is simply the filter's estimate of the friction torque, $T_{f}$, taken after enough time is given for the filter to converge to the associated fault level range.

\section{RESULTS}

Figures 5 and 6 below show time series for the friction torque estimation using the refined Kalman Filtering method on the healthy and PWM 25\% faulty development data, respectively. The ground-truth system friction, as derived in Appendix A, is indicated on the lower subplot by a dashed black line.

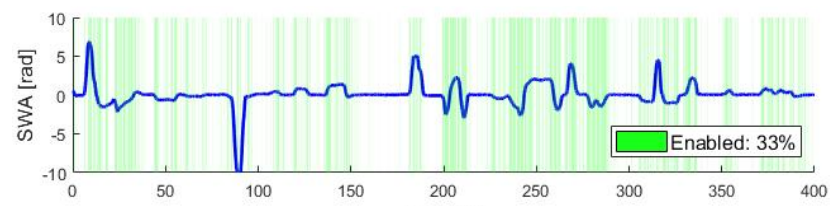

a) Steering profile

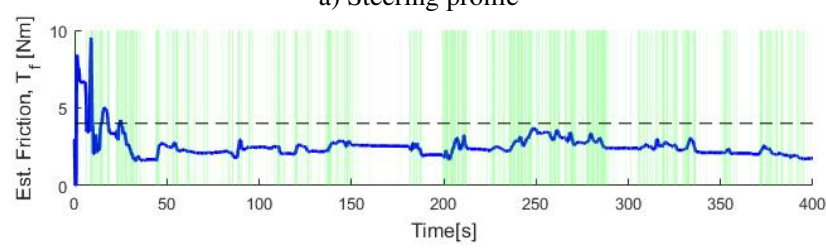

b) Estimated steering system friction torque

Figure 5. Friction estimation for healthy steering system

These results are generated with very simple enabling conditions that require the driver torque to exceed $0.5 \mathrm{Nm}$, the motor torque to be active in the same direction as the driver torque, and the steering wheel angle gradient to be less than $1 \mathrm{rad} / \mathrm{s}$ and larger than a minimum rate of $0.05 \mathrm{rad} / \mathrm{s}$. The first two conditions ensure the EPS system is active enough for the dynamic models to apply, while the third condition ensures the system is not changing too quickly for the filter to adapt. Whenever the enabling conditions are not met, the friction torque state estimate is not updated. All other states are updated at every step, regardless of enablement. The choice of covariance matrices and enablement rules for Eq. (5) and

\footnotetext{
${ }^{3}$ Some existing investigations on joint state-parameter estimation suggest starting with large VPN values and decreasing the value as time progresses. This does not apply to our case since we have a time-varying pseudoparameter that needs to be encouraged by a large VPN to continuously change. Reversely, the unknown constant parameter VPN needs to be kept constantly small to create the proper uncertainty channeling balance.
} 


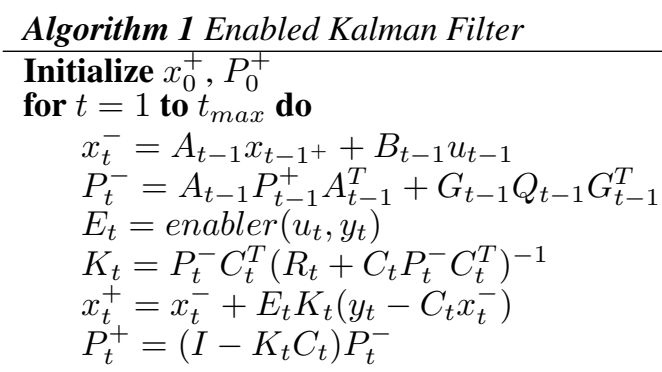

$\left.\begin{array}{r}\left.\text { (a priori estimate of } x_{t}\right) \\ \left.\text { (a priori estimate of } E\left[x_{t} x_{t}^{T}\right]\right) \\ (\text { Enabling matrix) } \\ (\text { Kalman gain) } \\ \left.\text { (a posteriori estimate of } x_{t}\right) \\ \left.\text { (a posteriori estimate of } E_{t}\left[x_{t} x_{t}^{T}\right]\right)\end{array}\right\} \quad$ Prediction step

Table 3. Enabled Kalman Filter summarized in Algorithm 1.

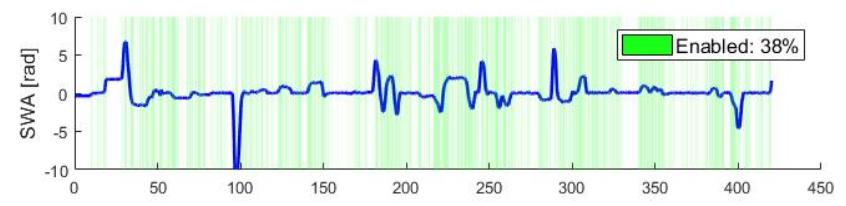

a) Steering profile

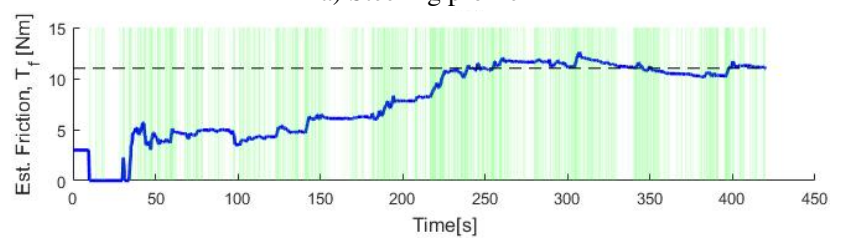

b) Estimated steering system friction torque

Figure 6. Friction estimation for faulty steering (25\% PWM)

Algorithm 1 are summarized below.

$$
\begin{aligned}
& Q_{t}=E\left[w_{t} w_{t}^{T}\right]=\left[\begin{array}{ccccc}
0.01 & 0 & 0 & 0 & 0 \\
0 & 0.01 & 0 & 0 & 0 \\
0 & 0 & 0.1 & 0 & 0 \\
0 & 0 & 0 & 1 & 0 \\
0 & 0 & 0 & 0 & 0.001
\end{array}\right], \\
& R_{t}=E\left[v_{t} v_{t}^{T}\right]=\left[\begin{array}{ccc}
10^{-4} & 0 & 0 \\
0 & 10^{-4} & 0 \\
0 & 0 & 10^{-4}
\end{array}\right] \\
& E_{t}=\left[\begin{array}{ccccc}
1 & 0 & 0 & 0 & 0 \\
0 & 1 & 0 & 0 & 0 \\
0 & 0 & 1 & 0 & 0 \\
0 & 0 & 0 & 1 & 0 \\
0 & 0 & 0 & 0 & 1_{\{\text {enabled }\}}
\end{array}\right]
\end{aligned}
$$

where

$$
1_{\{\text {enabled }\}}= \begin{cases}1 & \text { if enabled }=1 \\ 0 & \text { if enabled }=0\end{cases}
$$

and enabled is the result of the logical computation $\left(\left|T_{d}\right|>\right.$ $0.5 \mathrm{Nm})$ AND $\left(\operatorname{sign}\left(T_{d}\right)=\operatorname{sign}\left(T_{m}\right)\right)$ AND $(0.05<|\dot{\theta}|<$
$0.1 \mathrm{rads})$. Note that the filter requires about 50 seconds (5, 000 samples) of enabled data (or, considering that the enabling conditions are met about a third of the time, about 150 seconds of total data) to converge when initialized at the nominal value of $3 \mathrm{Nm}$. The filter output from the first 50 seconds of enabled data is therefore rejected when assessing the system health.

Figure 7 shows the distributions (normalized histograms) of friction estimates on the entire data set (i.e., combined development and test data) for healthy and PWM 25\% faulty. Comparing with Figure 3, it is clear that the refined algorithm

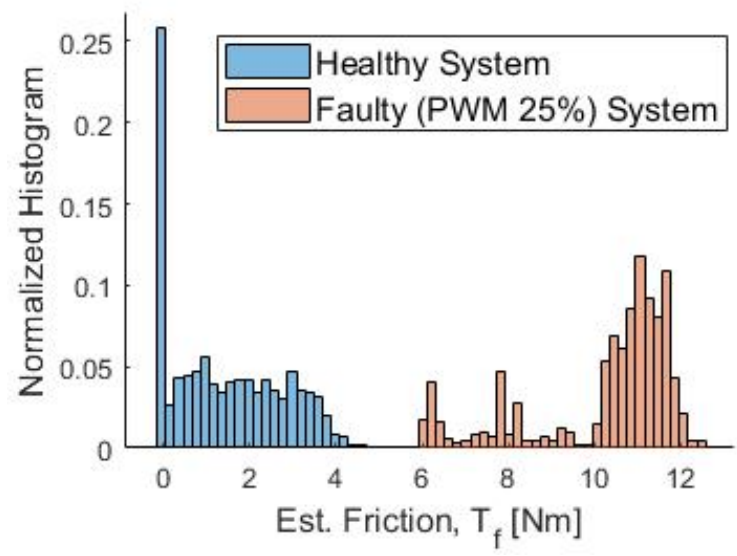

Figure 7. Distributions of friction estimates for healthy vs faulty (PWM 25\%).

exhibits superior performance to the baseline algorithm.

Figure 8 shows the performance of the refined friction estimation on all data in the development and test sets. The box edges show one standard deviation from the mean, and the whiskers show two standard deviations from the mean. Each health state has a friction estimate standard deviation between 1.5 and $2.2 \mathrm{Nm}$. Although there is a tendency to underestimate the ground-truth friction, this algorithm offers excellent performance in the task of classifying the levels of friction between a nominal $(4 \mathrm{Nm})$ and mildly faulted $(11-14 \mathrm{Nm})$ steering system. 


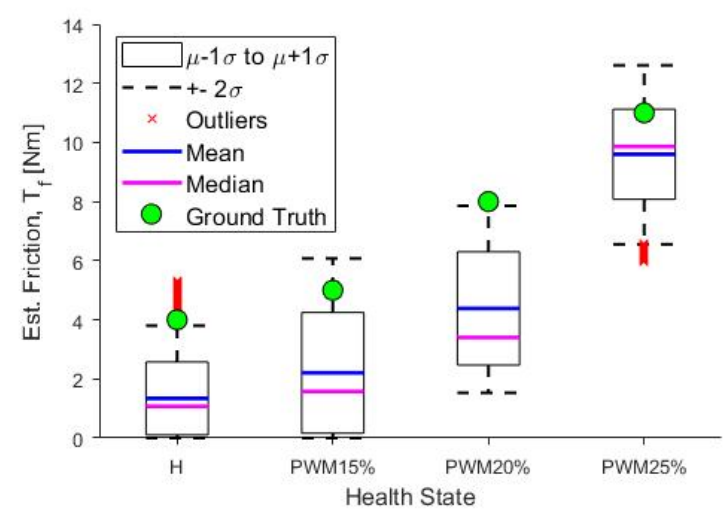

Figure 8. Box plots of Kalman Filter friction estimates on all data (development and test sets).

Figure 9 shows the distributions (normalized histograms) of friction estimates for healthy and PWM $25 \%$ faulty with different tire types. The results show that the refined algorithm

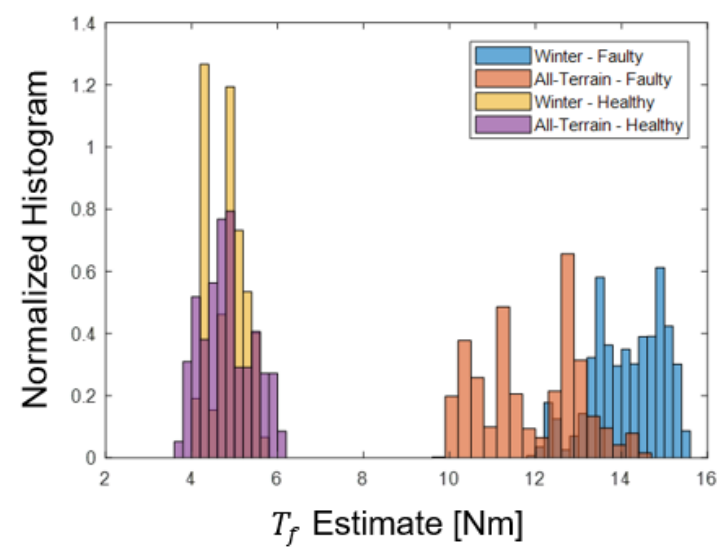

Figure 9. Distributions of friction estimates for healthy vs faulty (PWM 25\%) with different tire types.

is sensitive to some noise factors such as tire type when used to estimate the fault level. The estimation error induced on the fault level, however, is significantly below the requirement for separating a healthy system from a faulty one. As a result, the refined algorithm can still serve as a robust healthyfaulty classifier. Specifically, using the friction estimation algorithm, detection of increased friction can be, for example, achieved by setting a threshold and testing whether the estimated friction exceeds the threshold for a certain amount of time in a fixed interval length. We leave statistical analyses and selections of these tuning parameters for future follow-up work.

\section{Conclusions}

Data collected from a test vehicle under normal driving with different speeds, steering maneuvers, tire types, and levels of steering friction are used to validate and refine the friction detection algorithms proposed in (Lin \& Ghoneim, 2016). After a series of refinements described in a step-by-step fashion, the friction detection algorithm was enhanced to not only distinguish friction increase faults from the healthy system with maximum accuracy, but also estimate the friction in the steering system with a standard deviation of $2 \mathrm{Nm}$. This refined algorithm meets the objective of detecting friction increases before the 11-14 Nm level at which they become detectable to a driver. Future work can involve investigating the use of unscented Kalman filters or particle filters, projection techniques and systematic adaptation and tuning of noise covariances. The objectives are improving friction estimation accuracy, achieving finer separability of fault levels and decreasing required calibration effort.

\section{ACKNOWLEDGMENT}

The authors would like to thank Mr. Tim Romelhardt and Mr. James Di Donato for their support in test drives and data collection, and Dr. Yilu Zhang for his feedback on a preliminary version of this paper.

\section{REFERENCES}

Amberkar, S., Kushion, M., Eschtruth, K., \& Bolourchi, F. (2000). Diagnostic development for an electric power steering system. In SAE 2000 World Congress.

Badawy, A. A., Bolourchi, F., \& Gaut, S. K. (1997). Esteer system redefines steering technology. Automotive Engineering, 105(9), 15-18.

Denton, T. (2004). Automobile electrical and electronic systems. Routledge.

Huang, X., \& Wang, J. (2013). Identification of ground vehicle steering system backlash. Journal of Dynamic Systems, Measurement, and Control, 135(1).

Jeong, Y., Kim, K., Yoon, J., Chong, H., Ko, B., \& Yi, K. (2015). Vehicle sensor and actuator fault detection algorithm for automated vehicles. In Proceedings of the 2015 IEEE Intelligent Vehicles Symposium.

Ji, X., Ge, J., \& Tian, H. (2013). Reliability improvement of electric power steering system based on ISO 26262. In Proceedings of the 2013 International Conference on Quality, Reliability, Risk, Maintenance, and Safety Engineering.

Lawson, M., \& Chen, X. (2008). Fault tolerant control for an electric power steering system. In Proceedings of the 2008 IEEE Multi-conference on Systems and Control.

Lee, J., Lee, H., Kim, J., \& Jeong, J. (2007). Model-based fault detection and isolation for electric power steering system. In Proceedings of the 2007 International Con- 
ference on Control, Automation and Systems.

Lin, W.-C., \& Du, X. (2018). Prognosis of power connector disconnect and high resistance faults. In Proceedings of the 2018 IEEE International Conference on Prognostics and Health Management.

Lin, W.-C., \& Ghoneim, Y. A. (2016). Model-based fault diagnosis and prognosis for electric power steering systems. In Proceedings of the 2016 IEEE International Conference on Prognostics and Health Management.

Mohtat, A., Ghaffari Toiserkan, K., \& Kövecses, J. (2011). Recursive state-parameter estimation of haptic robotic systems. In Proceedings of the 2011 IEEE/RSJ International Conference on Intelligent Robots and Systems.

Mosquera Alonso, A. (2019). State- and parameter estimation for spacecraft with flexible appendages using unscented kalman filters . Luleå University of Technology.

Wan, E. A., \& van der Merwe, R. (2001). Kalman filter and neural networks. In S. Haykin (Ed.), (chap. The Unscented Kalman Filter). John Wiley \& Sons, Inc.

\section{BIOGRAPHIES}

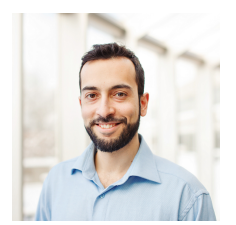

Arash Mohtat received his B.Sc. and M.Sc. from Univ. of Tehran in 2007 and 2009; and, his Ph.D. from McGill University in 2014. His theses to fulfill these degrees were focused on vibration control, smart materials and haptic robotic systems. From 2015 to early 2017 , he was a postdoctoral fellow at McGill University working on multi-modal human-computer interfaces as well as a research project that spun off into Haply Robotics: a start up with the mission of democratizing haptics and robotics technologies by making them opensource and scalable. From 2017 to 2020 , Arash has been with the Canadian Technical Center of General Motors leading a number of advanced technical projects in the vehicle health management domain for different actuation and perception systems. Arash has very diverse research interests with a key central theme: mechatronic systems with different levels of intelligence and autonomy (with and without the human in the loop). As a researcher/engineer with entrepreneurial enthusiasm, he has several peer-reviewed publications and pending US Patents.

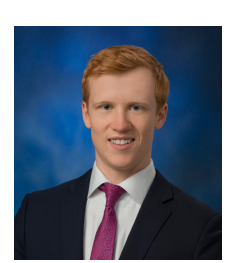

Graeme Garner received the B.S.E. from Queen's University, Ontario, Canada, in 2018, where he studied in a dual program of Applied Mathematics and Mechanical Engineering. He is the recipient of the J.B. Stirling Gold Medal for graduating with the highest academic standing in his class and the Keyser prize for his research project on adapting Q-learning to decentralized stochastic control problems. His diverse professional background includes developing oil production forecasting algorithms at the Alberta Energy Regulator and studying automated trading strategies at the Canadian Imperial Bank of Commerce. He is currently at the Canadian Technical Center of General Motors, where he develops prognostics algorithms for vehicle hardware. His research interests are in machine learning and control theory, and their intersection in intelligent vehicle systems.

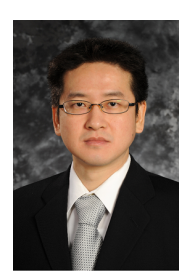

Wen-Chiao Lin received the M.S.E. and Ph.D. degrees in Electrical Engineering: Systems both from University of Michigan, Ann Arbor, MI. From 2007 to 2013 he was with Idaho National Laboratory, where he participated in research projects on developing advanced online anomaly detection and control technologies for large facilities, in which large amounts of sensors are deployed and may be unreliable or subject to cyber attacks. He is currently with General Motors Global R\&D, where he develops fault diagnosis, prognosis, and mitigation algorithms as well as fault tolerant control strategies for vehicle systems. His research interests are in the areas of systems science and control theory and their applications to engineering systems. He is the author or co-author of 30 peerreviewed publications and more than 20 issued or pending US Patents.

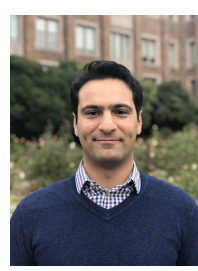

Naser Mehrabi received his M.Sc. in Mechanical Engineering from K. N. Toosi University of Technology, Iran; and, his Ph.D. in Systems Design Engineering from University of Waterloo, Ontario, Canada, in 2014. From 2014 to 2017, he was a postdoctoral fellow with Motion Research Group where he led research on biomechanical and neuromusculoskeletal modeling of human movement. From 2017 to 2018, he was a research fellow at University of Washington, where he developed computational models of human movements in order to study the effect of neuromuscular disorders such as cerebral palsy and stroke on the human motor control system. Naser longstanding expertise involves the modeling and control of multibody dynamic systems including vehicles, humans, and biomechatronic systems. Naser is currently with the Canadian Technical Center of General Motors leading advanced technical projects in the area of extreme vehicle dynamics control and estimation. Naser has published 12 peer-reviewed journal publications and has several pending US Patents.

\section{APPENDix A: Friction InTROduCED FOR TEST VE- HICLE}

Since there is no mapping between the PWM of the clutch brake used to inject friction faults and the corresponding friction increase in $\mathrm{Nm}$, the increase was estimated experimentally using a lock-to-lock test. In this test, the steering wheel is rotated clockwise from the neutral position $\left(0^{\circ}\right)$ to the lock point $\left(-570^{\circ}\right)$, then rotated counter-clockwise to the other lock point $\left(+570^{\circ}\right)$, and finally returned to neutral. A work balance over this loop yields the following estimate for the friction force:

$$
\begin{aligned}
& W_{\text {friction }}=W_{\text {driver }}+W_{\text {motor }}-\Delta K E-W_{\text {viscous }} \\
& \int T_{f} \operatorname{sign}(\dot{\theta}) d \theta= \\
& \quad \int\left(T_{d}+n T_{m}\right) d \theta-\frac{J_{e q}}{2}\left(\dot{\theta}_{2}^{2}-\dot{\theta}_{1}^{2}\right)-B_{e q} \int \dot{\theta} d \theta, \\
& T_{f}=\frac{\int\left(T_{d}+n T_{m}\right) d \theta-\frac{J_{e q}}{2}\left(\dot{\theta}_{2}^{2}-\dot{\theta}_{1}^{2}\right)-B_{e q} \int \dot{\theta} d \theta}{\int \operatorname{sign}(\dot{\theta}) d \theta}
\end{aligned}
$$




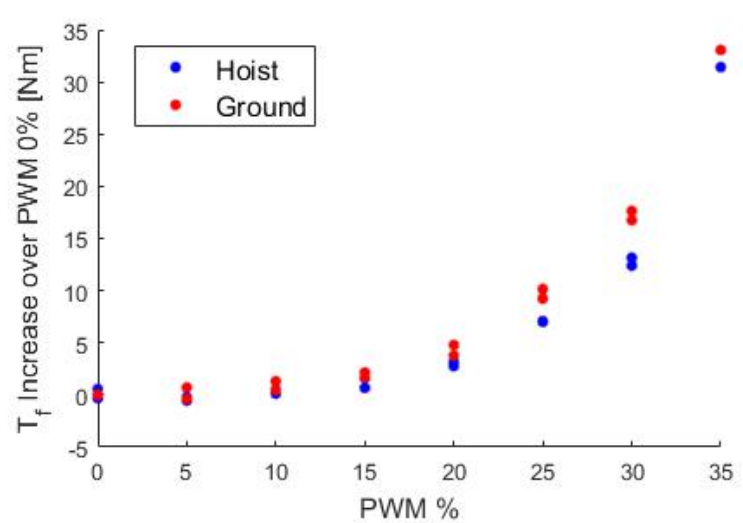

Figure 10. Estimated friction increase from clutch brake.

In the above equations, $K E$ is kinetic energy, and all other variables are as defined in Eq. (2) (Section 4.1).

Data was collected at PWM increments from $0 \%$ to $35 \%$ at steps of $5 \%$, with two lock-to-lock tests for each loop. The experiment was performed with the vehicle on a hoist and while stationary on the ground. The hoisted vehicle only experiences internal friction, whereas the ground vehicle also experiences scrub friction from the interaction of the tires and asphalt. To normalize these two experiments, the estimated increase in friction from modulating the clutch brake is calculated by subtracting the average friction for the two loops at $0 \%$ PWM from the estimated friction for every other experimental PWM. The results can be seen in Figure 10. The estimations shown in Figure 10 are presented in Table 1 as the range of injected torque from the clutch brake.

\section{APPENDIX B: Discussion ON Linearization}

The joint state-parameter model, see Eqs. (5) and (7), includes two nonlinear terms in its second row. Let us rewrite that second row individually, excluding the $B_{t} u_{t}$ and $G_{t} u_{t}$ terms, and ignoring the ${ }^{*}$ superscripts, as follows:

$$
\dot{\theta}_{t+1}=e^{-\tau} \dot{\theta}_{t}-\beta \rho\left(\theta_{t}\right) M_{z, t}-\beta \operatorname{sign}\left(\dot{\theta}_{t}\right) T_{f, t},
$$

where $\tau=d t B_{e q} / J_{e q}$. Adopting the convention $\left.X_{t}\right|_{t=t^{*}}=$ $X^{*}$ for any state variable $X_{t}$ evaluated at $t=t^{*}$, let us lin- earize the above equation around the operation point $t=t^{*}$. This yields

$$
\begin{aligned}
\dot{\theta}_{t+1}= & e^{-\tau} \dot{\theta}_{t}-\beta \rho\left(\theta^{*}\right) M_{z, t}-\beta \operatorname{sign}\left(\dot{\theta}^{*}\right) T_{f, t} \\
& -\beta \rho^{\prime}\left(\theta^{*}\right) M_{z}^{*}\left(\theta_{t}-\theta^{*}\right)-\beta \delta\left(\dot{\theta}^{*}\right) T_{f}^{*}\left(\dot{\theta}_{t}-\dot{\theta}^{*}\right),
\end{aligned}
$$

where $\rho^{\prime}=d \rho / d \theta$ and $\delta(X)$ is the Dirac's delta function (the analytical derivative of the sign function which is singular at zero). Now if we can ignore the last two terms of the above equation, we will legitimately return to the original linearized form represented by Eqs. (5) and (7). The rationale behind ignoring the first one of those two is that $\rho(\theta)$ is the road wheel angle ratio that can be assumed to be relatively constant in this context, i.e. $\rho^{\prime}$ is small. The rationale behind ignoring the other is that the Dirac's delta is zero everywhere but in the origin (the singularity point). We avoid the singularity by a judicious selection of the enabling conditions described in Section 5, specifically $|\dot{\theta}|>0.05$.

Last but not least, it is important to clarify what the operation point marked by the ${ }^{*}$ superscript is. One option is to use $\theta^{*}$ and $\dot{\theta}^{*}$ as available from the latest direct measurements for the (a priori) prediction step (see Table 3). Another alternative is to use the posteriori estimates of the angle and angular rate from the previous step. Both options lead to comparable results in our application. The former creates a smaller estimation error from ground truth, hence was chosen in this paper with results reported in Section 5. The latter, on the other hand, is more common in extended Kalman filtering (EKF) formulations. For us, it leads to larger mean absolute estimation errors in friction but tighter distributions of estimations around each fault level. So either of the options can be utilized depending on the relative importance of friction level estimation accuracy compared to separability of fault levels. Interestingly, the performance difference between the above two options implies that linearization achieves sub-optimal performance. Methods such as unscented Kalman filtering remove the necessity for linearization and its artifacts all together. And thus should be able to achieve the optimal estimation accuracy and separability simultaneously. 